\title{
APOIO EMOCIONAL REALIZADO POR ENFERMEIRO AO PACIENTE OSTOMIZADO
}

\author{
| Marilei Tavares e Souza1; Adriana Moraes²; Carlos Balbino3 ${ }^{3}$ Zenith Silvino ${ }^{4}$; Cláudia Tavares ${ }^{5}$; Joanir Passos ${ }^{6} \mid$
}

\section{RESUMO}

CONTEXTO: A existência de ostomia altera a imagem corporal do paciente, despertando vergonha e medo da rejeição social. O indivíduo passa a viver uma nova realidade, passando a mobililizar sentimentos e emoções negativas, interferindo em sua saúde mental. OBJETIVO: O estudo objetiva identificar reações causadas pela ostomia, medidas adotadas a partir do desconforto percebido pelo paciente ostomizado e a importância do apoio emocional proporcionado pela enfermagem.

METODOLOGIA: Pesquisa de abordagem qualitativa, realizada com 33 pacientes ostomizados, atendidos em uma Associação na cidade de Mendes - RJ. Os dados obtidos por meio de preenchimento de formulário foram submetidos à análise de conteúdo. Com aprovação do CEP, atendendo aos requisitos da Resolução 466/12 do CNS/MS.

RESULTADOS: As principias alterações constatadas na vida dos pacientes ostomizados estão relacionadas à vida social, imagem corporal, perda do controle sobre o corpo e sobre as emoções. Dentre as principais reações e sentimentos causados pela ostomia, destacam-se: vergonha, constrangimento, medo diante da nova situação, mal estar com odor, limitação e discriminação.

CONCLUSÕES: Ações de enfermagem necessitam ser sistematizadas para melhor atender as necessidades do paciente ostomizado. Para que ocorra amenização dos incômodos identificados, ressalta-se a importância de sistematização das intervenções de enfermagem e a adoção de medidas de apoio emocional por parte dos enfermeiros.

\section{PALAVRAS-CHAVE: Enfermagem; Ostomia; Emoções em saúde}

\section{RESUMEN}

\section{“Apoyo emocional realizado por la enfermera delestomía a pa-} ciente"

CONTEXTO: La existencia de estomía altera la imagen corporal del paciente, despertando la vergüenza y el miedo al rechazo social. El individuo comienza a vivir una nueva realidad comenzó a influir en los sentimientos y las emociones negativas, lo que afecta su salud mental. OBJETIVO: El estudio tiene como objetivo identificar las reacciones causadas por la estomía, medidas tomadas desde el malestar percibido por el paciente de estomía y la importancia del apoyo emocional proporcionado por el personal de enfermería.

METODOLOGÍA: La investigación cualitativa realizada con 33 pacientes con estomía, se reunieron en una asociación en la ciudad de Mendes - RJ. Los datos obtenidos a través de la forma de llenado se sometieron a análisis de contenido. Aprobado por el CEP, el cumplimiento de los requisitos de la CNS / MS Resolución 466/12.

RESULTADOS: Los principales cambios observados en las vidas de los pacientes con estomía están relacionados con la vida social, la imagen corporal, la pérdida de control sobre el cuerpo y las emociones. Las principales reacciones y sentimientos causados por la estomía en especial: la vergüenza, la vergüenza, el miedo ante la nueva situación, malestar con olor, limitación o discriminación.

CONCLUSIONES: Acciones de enfermería deben ser sistematizados para satisfacer mejor las necesidades del paciente con estomía. Para disminuir la problemática identificada, se hace hincapié en la importancia de la sistematización de las intervenciones de enfermería y la adopción de medidas de apoyo emocional por las enfermeras.

\section{DESCRIPTORES: Enfermería; Estomía; Emociones salud}

\section{ABSTRACT \\ "Emotional support provided by the nurse to the ostomized pa- tient"}

BACKGROUND: The presence of ostomy alters the patient's body image arousing shame and fear of social rejection. The individual begins to live a new reality through negative feelings and emotions, which could affect their mental health.

AIM: The study aims to identify reactions caused by ostomy, measures taken based on the discomfort perceived by the ostomized patient, and the importance of emotional support provided by the nursing staff.

METHODS: This was a qualitative research conducted with 33 ostomized patients treated at an Association in the city of Mendes - RJ. The data obtained through forms were subjected to content analysis. The study was approved by the CEP in compliance with requirements of the CNS/MS 466/12 Resolution.

RESULTS: The major alterations observed in the life of ostomized patients are related to social life, body image, and loss of control over body and emotions. The main reactions and feelings caused by ostomy are: shame, embarrassment, fear in the face of a new situation, malaise with odor, and limitation or discrimination.

CONCLUSIONS: Nursing actions need to be systematized to better meet the needsofostomized patients. The importance of systematization in nursing interventions and adoption of emotional support measures is emphasized to ameliorate identified discomforts.

\section{KEYWORDS: Nursing; Ostomy; Emotions in health}

Submetido em 22-02-2016

Aceite em 30-05-2016

\footnotetext{
1 Mestre; Doutoranda do Programa de Pós-Graduação em Enfermagem e Biociências da Universidade Federal do Estado do Rio de Janeiro; Professora do Curso de Enfermagem na Universidade Severino Sombra,Rua Xavier Sigaud, Rio de Janeiro, Brasil, marileimts@hotmail.com

2 Enfermeira no Hospital Nelson Salles, 26650-000 Rio de Janeiro, Brasil, adriana@frontincom.br

3 Mestre em Enfermagem Assistencial pela Universidade Federal Fluminense; Professor na Universidade Severino Sombra, Brasil, carlosmbalbino@hotmail.com 4 Doutora em Enfermagem pela Universidade Federal do Rio de Janeiro;Titular na Universidade Federal Fluminense, Niterói - RJ, Brasil, zenithrosa@terra.com.br 5 Pós-Doutora pela Universidade de São Paulo; Doutora em Enfermagem; Professora Titular na Universidade Federal Fluminense, Brasil, claudiamarauff@gmail.com 6 Doutora em Enfermagem pela Universidade de São Paulo; Professor Associado na Universidade Federal do Estado do Rio de Janeiro, Brasil, joppassos@hotmail.com
} 


\section{INTRODUÇÃO}

Portadores de estoma possuem características comuns que os unem em um grupo especial, com necessidades e reações próprias implícitas à sua identidade e subjetividade. Assim, a resposta à problemática causada pela abertura do estoma guarda relação com as condições pessoais de cada um, bem como com as variações externas, como qualidade do suporte familiar, financeiro e assistencial recebidos em todas as fases do tratamento cirúrgico gerador de estoma.

A palavra estoma tem origem grega e exprime a ideia de boca ou abertura do segmento cólico na parede abdominal, visando o desvio do conteúdo fecal para o meio externo (Violin, 2008). Os estomas podem ser implantados em diversas áreas do corpo que necessitam de um tratamento, e auxílio no melhor funcionamento orgânico. A presença de ostomia altera a imagem corporal do paciente, podendo despertar sentimentos de vergonha e medo da rejeição social (Barbutti, Silva e Abreu, 2008).

$\mathrm{O}$ indivíduo que possui estoma passa a viver uma nova realidade, o que mobiliza sentimentos e emoções negativas, interferindo em sua saúde mental(Marques e Lopes, 2015).

Devemos considerar que é de suma importância o planejamento e implementação da assistência de enfermagem, que requer além dos cuidados físicos, ensinar ao paciente os cuidados de higiene e troca de bolsas de ostomia, incluindo planejamento da assistência ao período operatório com visitas e o pré-operatório, demarcação de estoma, preparo físico propriamente dito, integração das intervenções com a equipe do bloco cirúrgico. Requer ainda a retomada do ensino préoperatório para o autocuidado, envolvendo paciente/ família, visando à reabilitação e encaminhamento ao Programa de Ostomizados, que é mantido pelo serviço público, para aquisição dos dispositivos e seguimento ambulatorial (Barbutti, Silva e Abreu, 2008).

Articulando-se com os preceitos do Sistema Único de Saúde (SUS) no que se refere à divulgação do conceito de saúde como qualidade de vida e direito do cidadão, humanização do atendimento e desenvolvimento de atividades de promoção da saúde. Mas para que ocorra implementação do cuidado com ações humanizadoras, torna-se indispensável valorizar a dimensão subjetiva e social em todas as práticas de atenção (Souza, Passos e Tavares, 2015).
A complexidade da assistência de enfermagem a ser prestada nos remete à necessidade de compreender as modificações que ocorrem em sua vida, como estimular a autonomia, acolher os sofrimentos, resolver os problemas, estabelecer vínculos (Neves, Souza, Tavares e Vasconcelos, 2014). Muitas vezes, ao tentar conhecer os mecanismos produtores de doenças, os profissionais de saúde distanciam-se das relações com os seres humanos. O desenvolvimento de tecnologias de relacionamento pode ser um meio de restabelecer diálogos, acolher os sofrimentos, resolver os problemas, estabelecer vínculos e responsabilidades e estimular a autonomia dos usuários (Souza, Passos e Tavares, 2015).

O estudo tem por objetivo identificar reações causadas pela ostomia, medidas adotadas a partir do desconforto percebido pelo paciente ostomizado e a importância do apoio emocional proporcionado pela enfermagem.

\section{METODOLOGIA}

Trata-se de investigação do tipo exploratória de natureza qualitativa, cuja ênfase são as relações, representações, crenças, histórias, percepções e opiniões, produtos das interpretações que os humanos fazem a respeito de como vivem, constroem seus artefatos e a si mesmos, sentem e pensam (Minayo, 2010).

A pesquisa foi realizada na Associação dos Ostomizados localizada no Centro-Sul Fluminense, na cidade de Mendes, Rio de Janeiro. Elegeu-se como técnica de investigação, um formulário contendo: dados sócios demográficos, seguido das questões: quais incômodos têm por ser ostomizado? Que medida(s) adota $(\mathrm{m})$ para melhoria dos incômodos relatados? A coleta dos dados ocorreu entre março e abril de 2014. Foram participantes do estudo 33 pacientes ostomizados que fazem parte do Núcleo dos Ostomizados de Mendes.Foram selecionados para participar do estudoaqueles que atenderam aos critérios de inclusão da pesquisa: mais de seis meses de uso da bolsa de colostomia, tempo ideal para adaptação e participar do núcleo dos ostomizados do serviço referido. Critérios de exclusão: clientes com idade igual ou inferior a 18 anos de idade.

A análise dos dadosdeu-se a partir da Técnica de Análise de Conteúdo Categorial (Bardin, 2011). Na pré-análise selecionou-se o material, e, em seguida, realizou-se a leitura flutuante do mesmo, deixando-se impregnar pelo conteúdo, observou-se a dinâmica existente entre a questão norteadora inicial e as questões emergentes, complementando-as com a leitura de textos teóricos relacionados ao tema. 
Foi garantido o anonimato de cada participante do estudo, utilizou-se a letra "P" (participantes) seguida dos números 1 ao 33, atendendo às exigências da Resolução Resolução 466/12 do Conselho Nacional de Saúde, que dispõe sobre as normas e diretrizes regulamentadoras de pesquisas envolvendo os seres humanos (Ministério da Saúde do Brasil, 2012). O estudo foi aprovado pelo Comitê de Ética em Pesquisa (CEP) da Faculdade Sul Fluminense (FASF) de Volta Redonda, Rio de Janeiro, sob o número $01 / 2014$.

\section{RESULTADOS}

\section{Caracterização da Amostra}

Através dos resultados apresentados no Quadro 1 podemos verificar que em relação ao gênero $57.57 \%$ da amostra, o sexo feminino predomina.

Quadro 1 - Caracterização Quanto ao Gênero dos Participantes

\begin{tabular}{|c|c|c|}
\hline Gênero & Frequência & $\mathrm{f}(\%)$ \\
\hline Feminino & 19 & 57,57 \\
\hline Masculino & 14 & 42,42 \\
\hline TOTAL & 33 & $100 \%$ \\
\hline
\end{tabular}

A faixa etária da população estudada é apresentada no Quadro 2, no qual podemos observar que há predomínio de adultos e idosos, prevalecendo ida $\neg$ de superior aos 40 anos, seguida de aumento da incidência com o avanço da idade. Portanto, o envelhe-cimento apresenta-se como um fator de risco para o desenvolvimento da neoplasia e, conse $\neg$ quentemente, a possibilidade de realização do estoma, evidenciada através do predomínio da faixa etária de idosos, representando $66,66 \%$ da amostra.

Quadro 2 - Distribuição da Idade dos Participantes por Faixa Etária

\begin{tabular}{|c|c|c|}
\hline Idade (em anos) & Frequência & $\mathrm{f}(\%)$ \\
\hline $21 \mid---------31$ & 2 & 06,06 \\
\hline $31 \mid----------41$ & 2 & 06,06 \\
\hline $41 \mid---------51$ & 7 & 21.21 \\
\hline $51 \mid---------61$ & 8 & 24,24 \\
\hline $61 \mid---------71$ & 8 & 24,24 \\
\hline $71 \mid---------81$ & 5 & 15,15 \\
\hline $81 \mid---------91$ & 1 & 03,03 \\
\hline TOTAL & 33 & $100 \%$ \\
\hline
\end{tabular}

Os dados apresentados no Quadro 3 permitem visualizar o tipo de ostomias utilizadas pelos participantes do estudo, apontando maior frequência para colostomias, com 63,64 ( $n=21)$, seguido de ileostomias, com 30,30\% $(n=10)$.
Quadro 3 - Classificação dos Pacientes quanto ao Tipo de Ostomia

\begin{tabular}{|c|c|c|}
\hline Tipo de ostomia & Frequência & Percentual \\
\hline Colostomia & 21 & $63,64 \%$ \\
\hline Ileostomia & 10 & $30,30 \%$ \\
\hline Urostomia & 02 & $6,06 \%$ \\
\hline Total & 33 & $100.0 \%$ \\
\hline
\end{tabular}

\section{Principais Fatores que Causam Sofrimento na Vida dos Ostomizados}

Reações e sentimentos dos ostomizados.

As principias alterações constatadas na vida dos pacientes ostomizados estão relacionadas à vida social, imagem corporal, perda do controle sobre o corpo e sobre as emoções.

E dentre as principais reações e sentimentos causados pela ostomia, destacam-se: vergonha, constrangimento, medo diante da nova situação, mal-estar com odor, limitação e discriminação. Como pode ser destacada nas transcrições a seguir:

Como posso ir para casa, sem saber aonde vou conseguir a bolsa para sobreviver após a cirurgia? Será que um dia vou voltar a fazer o que fazia antes? Ter uma vida normal, contato com o público, vida social, família, amigos é difícil sem um bom equipamento. [P9] Por não ter uma bolsa adequada à pele fica machucada, assada, dura e ferida. A bolsa descola e as fezes caem, passamos constrangimentos na rua e em casa. Assim, não podemos sair de casa por não ter segurança. A vida sexual também fica difícil por causa da bolsa.[P10] [...] usava pano e ficava dentro de casa com vergonha de falar com o vizinho por causa do cheiro ruim[...]. [P16] Ser discriminado, falta de informação e bolsas de qualidade. Estar seguro para continuar a minha vida como antes. [P17]

Vários incômodos, como por exemplo viajar, participar de festas, dificuldade de lidar com a ostomia. [P26]

\section{Desconforto causado pela ostomia.}

Para pacientes portadores de ostomia, o desconforto ou incômodo, na maioria das vezes, está relacionado à falta de orientação sobre como usar a bolsa e sobre o autocuidado, além da falta de apoio emocional. Sendo este um dos fatores que contribuem para a boa adaptação ou não do ostomizado. O suporte emocional deve ser dado pela família, amigos e, sobretudo pelo enfermeiro, como se verifica nos depoimentos a seguir:

Sair do hospital sem saber como irá fazer para conseguir as bolsas coletoras, então, passa na sua cabeça: como vou viver com a ostomia sem bolsa adequada para levar uma vida melhor e digna na sociedade e na família? [P2] 
Vou a associação para obter as bolsas, lá eles ensinam como usar a bolsa para sair de casa, passear e ter contato com a sociedade. [P3]

Encaminham as pessoas para receber bolsas coletoras, para a vida ser normal e também orientam a família. [P25]

Ainda não encontrei nenhuma medida para amenizar o meu incômodo. [P26]

Tratar ostomizado com carinho. [P32]

\section{Apoio emocional prestado pela enfermagem.}

Para que a transmissão de afetividade se dê de forma concreta é necessário que o enfermeiro trabalhe junto ao paciente, aspectos práticos como o conhecimento sobre ostomia e a maneira como o paciente imagina a vida com a bolsa coletora.Esse suporte permitirá que o paciente expresse seus sentimentos e o enfermeiro tenha subsídios para possíveis intervenções, como pode ser percebido na transcrição a seguir:

Sair do centro cirúrgico sem saber o que fazer, por estar com um saco no abdome, e não saber o que fazer quando sair do hospital. [P9]

Desta forma, para que o paciente se sinta mais seguro, e possa falar sobre seus sentimentos e primeiras impressões acerca da ostomia, o apoio emocional prestado pela enfermagem deve ocorrer desde a primeira consulta.

Orientação, após a cirurgia, o paciente ostomizado, tem que sair do hospital com orientação, que tem que participar da associação, para ser reabilitado e saber que pode levar uma vida normal. [P2]

Como a enfermagem é a responsável pelas primeiras orientações sobre ostomia, deve reservar tempo para o suporte emocional tanto do paciente como para a família, apontando aspectos positivos do tratamento. Entender que dar apoio emocional implica, sobretudo, mostrar aspectos que possam amenizar o tratamento.

[...] pensar que pessoas estão passando pelo que eu passei. Sai do hospital e não tive um apoio, num tive aonde conseguir bolsas, passei vergonha, usei bolsa de mercado na barriga.[P1]

Como podemos perceber nas transcrições dos depoimentos dos participantes, o apoio emocional prestado pela enfermagem poderá se manifestar em atitudes como respeito, atenção, informação, segurança, afeto, incentivo, deixar claro para o paciente que está disponível para auxiliá-lo.

Sair do hospital sem saber como vou conseguir bolsas é desumano. Sair do hospital sem informações é falta de amor aos outros. [P24]
O depoimento a seguir destaca a necessidade de formação específica do profissional de saúde para atender às necessidades de cuidados dos pacientes ostomizados. O próprio paciente fala das deficiências da formação profissional nesse sentido, destacando a importância do preparo dos profissionais durante seu processo de formação.

Tem enfermeiro e também outros profissionais que desconhecem o caso e que nunca viram um estoma durante sua formação. Já passei por várias situações. Médicos, enfermeiros e outras autoridades teriam que se voltar mais para esse assunto, os ostomizados merecem mais atenção. [P9]

\section{DISCUSSÃO}

A partir da análise conjunta dos resultados referentes a caracterização da amostra, verifica-se que o resultado está em consonância com a estimativa da incidência de câncer no Brasil (Instituto Nacional de Câncer, [INCA], 2012).

Cabe ressaltar que a incidência de câncer no mundo cresceu $20 \%$ na última década. O impacto do câncer na população corresponderá a $80 \%$ entre os países em desenvolvimento, dos mais de 20 milhões de casos novos estimados para 2025. No Brasil, estima-se 576 mil casos novos para o 2016 (INCA, 2015).

A idade superior a 60 anos é descrita na literatura como um dos principais marcadores para a identificação de grupos de risco. A relevância deste fato acentua-se na constatação das projeções da Organização Mundial da Saúde (OMS) para 2025, que aponta o Brasil entre os 10 países do mundo com maior número de idosos. Mudança que tem relação com uma importante transição epidemiológica, representando significativo crescimento da demanda de serviços de saúde, acarretando tratamento de longa duração, recuperação mais lenta e intervenções com custos elevados. Ou seja, requer um sistema amplo, coordenado e contínuo de ações (Perez, 2008).

Importante ressaltar que a transição demográfica, que se caracteriza pelo envelhecimento populacional, é um processo que ocorre mundialmente, seguido de mudança epidemiológica apontada pela prevalência de doenças crônicas degenerativas, entre elas o câncer. Mais de $60 \%$ dos casos encontram-se na população idosa (Vieira e Marcon, 2008).

Hoje, o envelhecimento populacional é considerado um dos maiores desafios da saúde pública contemporânea. 
Um fenômeno que ocorreu inicialmente nos países desenvolvidos, mas vem acontecendo de forma mais intensa nos países em desenvolvimento, como é o caso do Brasil (Ramos, 2009).

Neste sentido, cabe destacar a importân $\neg$ cia do papel do enfermeiro, que deve favorecer o autocuidado e segurança do paciente como fator prepon $\neg$ derante em sua recuperação, bem como intensificar o trabalho junto à população adulta para a melhoria da qualidade de vida e diminuição dos agravos a sua saúde.

Quanto a classificação e tipo de ostomia, o resultado está em consonância com estudos já realizados, onde a ocorrência do evento está relacionada à causa que levou o sujeito a estomia, que na maioria das vezes se dá pela presença de câncer de intestino, seguido de obstrução intestinal (Santos, Bezerra, Bezerra e Paraguassú, 2007). Os tipos de ostomia podem variar, mas em todos os casos requer atenção e orientação adequada para evitar agravos e complicações referentes ao manuseio inadequado de sua ostomia. A participação do enfermeiro neste processo é fundamental para orientar e contribuir com o atendimento adequado, promovendo aos usuários um cuidado livre de riscos e orientado à promoção da sua saúde. Neste sentido, o enfermeiro exerce uma função de mediação, devendo auxiliar o ostomizado ou candidato a ostomia, a resolver seus problemas, incluindo apoio emocional no processo de adaptação e autonomia (Balbino, 2010).

Como o portador de ostomia inicia sua trajetória na internação hospitalar, com continuidade no retorno ao meio em que vive, é importante prestar a devida orientação quanto a sua nova condição, que inclui modificações físicas, biopsíquicas, sociais, econômicas e culturais. Assim, no retorno a sua rotina é travada uma luta constante, onde o enfermeiro deve oferecer todo o suporte tanto ao paciente quanto ao seu familiar, para que haja qualidade de vida para o paciente com ostomia.

Torna-se primordial um tempo interno para viver a dor da perda, reavaliar conceitos e buscar recursos de enfrentamento para se adaptar à nova vida (Barbutti, Silva e Abreu, 2008).

O desenvolvimento de intervenções efetivas direcionadas para a assistência integral durante o tratamento é considerado um desafio para a enfermagem. Requer estratégias relacionadas na reavaliação positiva, apoio emocional e suporte social, formas ativas e positivas que resultem na adaptação ao processo de enfrentamento do problema (Nunes e Souza, 2015).
No que diz respeito as reações e sentimentos, verificase a partir das transcrições dos depoimentos dos participantes do estudo que é preciso investir no trabalha emocional junto ao paciente ostomizado para que haja a reconstrução de sua autoimagem, sendo necessário apoia-lo a viver e elaborar o luto do corpo da antiga imagem corporal. A alteração da imagem corporal pode refletir negativamente na autoestima, no autoconceito, na sexualidade e, consequentemente, na identidade pessoal (Barbutti, Silva e Abreu, 2008).

É comum o acometimento de isolamento social, alteração da vida sexual e da vida laboral impostos pelo próprio paciente, em razão dos sentimentos de inutilidade, incapacidade, desprestígio e vergonha por se sentir diferente (Nascimento, Trindade, Luz e Santiago, 2011). O portador de estomia, muitas vezes, incorpora um estigma social, pois se sente desigual diante das outras pessoas, apresentando a preocupação de esconder a estomia até mesmo de seus familiares. Conviver com a estomia pode acarretar dificuldades no desenvolvimento de atividades de lazer e de convívio social (Caetano et al., 2014).

Quanto ao resultado relacionado ao deconforto causado pela ostomia, nos leva a refletir sobre a realidade vivida pelo portador de ostomia e o quanto é importante uma prática adequada.Pelo fato de o enfermeiro ter papel primordial junto ao paciente ostomizado, geralmente é ele quem dá as primeiras orientações sobre a ostomia, ou seja, sana as dúvidas, trabalha junto ao paciente nos cuidados básicos, incentiva a pessoa a olhar para si, pois alguns pacientes adiam esse momento e encontram dificuldade para isso.

A prática adequada da enfermagem é essencial e faz parte do processo de incorporação e integração psicológicas da ostomia. Assim, o apoio emocional incorporado à prática do enfermeiro favorece a interação e mediação com o paciente, tornando-o capaz de reconhecer ações que trazem segurança e tranquilidade emocionais, o que indica que os próprios pacientes podem encontrar em si mesmos, meios para educarem suas emoções (Fonseca e Tavares, 2015).

Apoio emocional constitui ação profissional com intuito de oferecer ao paciente amparo, segurança, suporte, orientações e proteção. De acordo com Sluzki (1997), refere-se a intercâmbios que conotam uma atitude emocional positiva, clima de compreensão, simpatia, empatia e estímulo; é o poder contar com a ressonância e a boa vontade do outro. 


\section{IMPLICAÇÕES PARA A PRÁTICA CLÍNICA}

No que se refere ao apoio emocional prestado pela enfermagem ao paciente ostomizado, o estudo demonstrou a relevância em prestar orientações adequadas sobre a nova realidade a ser vivenciada, auxiliando no processo de compreensão e desenvolvimento da capacidade de trabalhar suas emoções. $\mathrm{O}$ enfermeiro deve possuir subsídios que favoreçam o cuidado efetivo dos pacientes ostomizados e seus familiares.

\section{REFERÊNCIAS BIBLIOGRÁFICAS}

Balbino, C.M. (2010). A gerência do cuidado de enfermagem na implantação de um espaço de cuidar em saúde à comunidade escolar (Dissertação de Mestrado). Niterói, Rio de Janeiro: Universidade Federal Fluminense.

Barbutti, R.C.S, Silva, M.C.P, e Abreu, M.A.L. (2008). Ostomia, uma difícil adaptação. Revista da Sociedade Brasileira dePsicologia Hospitalar, 11(2), 27-39. Disponível em http://pepsic.bvsalud.org/pdf/rsbph/v11n2/ v11n2a04.pdf

Bardin, L. (2011). Análise de conteúdo. Lisboa: Edições 70.

Ministério da Saúde do Brasil. (2012). Resolução n466, de 12 de dezembro de 2012. Diretrizes e normas regulamentadoras de pesquisas envolvendo seres humanos. Brasília: Conselho Nacional de Saúde.

Caetano, C.M., Beuter, M., Jacobi, C.S., Mistura, C., Rosa, B.V.C., e Seiffert, M.A. (2014). O cuidado à saúde de indivíduos com estomias. Revista Brasileira de Ciências da Saúde, 12(39), 59-65.

Fonseca, P.N., e Tavares, C.M.M. (2015). O manejo das emoções dos coordenadores em transplantes na realização da entrevista familiar para doação de órgãos. Revista Portuguesa de Enfermagem de Saúde Mental (Ed. Esp. 2), 39-44.

Instituto Nacional de Câncer.Coordenação de Prevenção e Vigilância.(2015). Dia Nacional do Câncer. Estimativa 2016: Incidência de câncer no Brasil. Rio de Janeiro: Instituto Nacional de Câncer. Disponível em http://www.inca.gov.br/wcm/dncc/2015/dados-apresentados.pdf
Instituto Nacional do Câncer. Ministério da Saúde. (2012). Estimativa 2012: Incidência de câncer no Brasil. Rio de Janeiro: Instituto Nacional de Câncer. Disponível em http://www. inca.gov.br/estimativa/2012/index. asp?ID=5

Marques, M.F., e Lopes, M.J. (2015). O cuidador familiar no olhar da pessoa com depressão. Revista Portuguesa de Enfermagem de Saúde Mental (Ed. Esp. 2), 51-56.

Minayo, M.C.S. (2010). O desafio do conhecimento: Pesquisa qualitativa em saúde. São Paulo: Hucitec.

Nascimento, C.M.S, Trindade, G.L.B, Luz, M.H.B.A., e Santiago, R.F. (2011). Vivência do paciente estomizado: Uma contribuição para a assistência de enfermagem. Texto \&Contexto Enfermagem, 20(3), 557-564.

Neves, E.S., Souza, M.M.T, Tavares, C.M., e Vasconcelos, C.B.S. (2014). The working process of health caregivers who work in therapeutic residences. Revista PróUniverSUS, 5(1), 21-26.

Nunes, N.C.F., e Souza, M.M.T. (2015). Reflexões sobre a repercussão do câncer de mama para o binômio cliente-família. Revista Pró-UniverSUS, 6(3), 23-25.

Perez, M. (2008). A população idosa e o uso de serviços de saúde. Revista do Hospital Universitário Pedro Ernesto, 7(8), 30-37.

Ramos, L. R.. (2009). Saúde pública e envelhecimento: O paradigma da capacidade funcional. Boletim do Instituto de Saúde, 47,40-41.

Santos, C.H.M., Bezerra, M.M., Bezerra, F.M.R.M., e Paraguassú, B. R. (2007). Perfil do paciente ostomizado e complicações relacionadas ao estoma. Revista Brasileira de Coloproctologia, 27(1), 16-19.

Sluzki, C. E. (1997). A rede social na prática sistêmica: Alternativas terapêuticas. São Paulo:Casa do Psicólogo.

Souza, M.M.T, Passos, J.P., e Tavares, C.M.M. (2015). Suffering and precarious ness at work in nursing. JournalofResearch: Fundamental Care,7(1), 2072-2082. 
Vieira, M.C.U., Marcon, S.S. (2008). Significados do processo de adoecer: $\mathrm{O}$ que pensam cuidadoras principais de idosos portadores de câncer. Revista da Escolade Enfermagem da USP, 42(4), 752-760.
Violin, M.R., Mathias, T.A.F, e Uchimura, T.T. (2008). Perfil de clientes colostomizados inscritos em programa de atenção aos estomizados. Revista Eletrônica de Enfermagem, 10(4), 924-932.

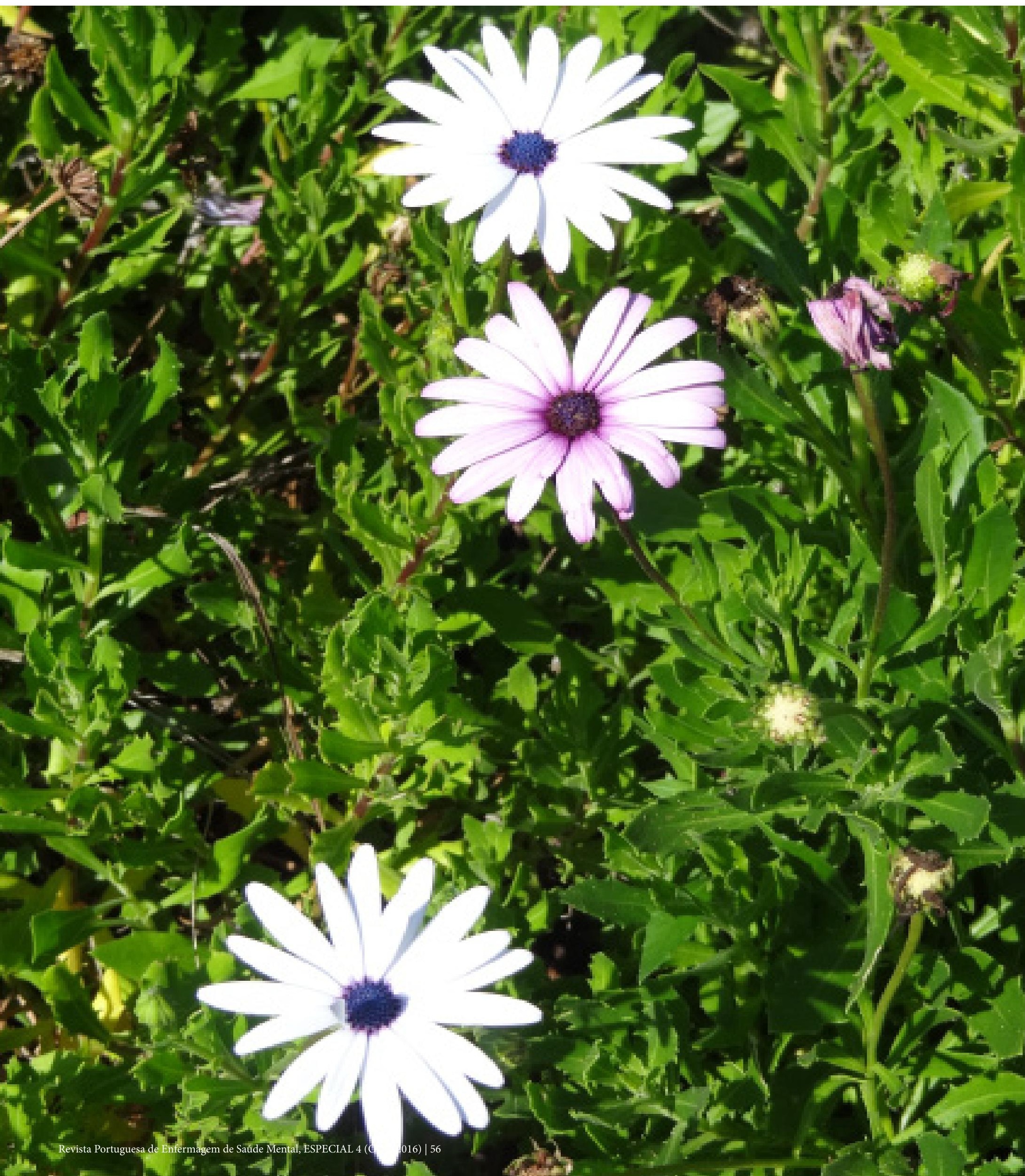

